retired from Brunel University in Uxbridge, $\mathrm{UK}$, was among the first people invited in those early days and served on the WIF's board. But he became quickly disillusioned, he says, and has had nothing to do with the WIF since. "My impression was that Hill had lots of big ideas, but few results," says Ralph, adding that "Hill did seem to be able to attract people to go on headed notepaper by the bucketload".

The foundation's main current activity is providing scientific advice on sustainable development to governments worldwide, says Hill. He seems to be a one-man Davos conference, dropping names of ministers, ambassadors and heads of states that he has met, noting nonchalantly that he had a chat with Mikhail Gorbachev recently at a Nobel peace summit.

The foundation does have at least one project in the public domain. Baehr is testing a Vietnamese herbal remedy for heroin addiction - a project he began at the United Nations (see Nature 433, 568-569; 2005). The WIF has helped to open doors, says Ludger Wessjohann, an organic chemist at the Leibniz Institute of Plant Biochemistry in Weinberg, Germany, who is involved in the project. Hill says the WIF also has projects spanning from avian flu, to bringing solar electricity to rural Africa, to building research centres in Kazakhstan and Kyrgyzstan. But it is difficult to find out more, because the details, says Hill, are "confidential".

"It is a mysterious organization," says Ian Pearson, a WIF fellow and a former futurologist at British Telecom, "I was invited to join a couple of years ago and allowed them to re-use one of my articles for their web site, and that is the last contact I had... It says at the outset that it works in secrecy, so of course there is no way of knowing whether it is extremely active or totally defunct." Pearson adds that he is likely to remove the WIF honours from his website.

Declan Butler

curricula, she says. "Creation science is alive and well and appealing to a substantial minority of the American public."

Miller, himself an evangelical Christian, says that scientists must be careful when responding to the launch of $A R J$. Taking too strong a stand against the journal will fuel creationists' accusations of scientific 'bias' against religion, he argues. Researchers should instead try to educate non-scientists about the scientific process, he says.

Geoff Brumfiel

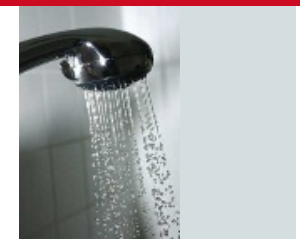

MUSE: THE MATHS BEHIND GROUP SHOWERS Read Nature's online columns. www.nature.com/news/ opinion/index.html

\title{
Huge crystal baffles chemists
}

A giant molecular bauble, by far the largest single-molecule crystal ever made, is confounding chemists. Involving almost 500 silver atoms, the crystals are so large and complex that their creators cannot figure out their structure.

Each of the 30 crystals made by Dieter Fenske of the University of Karlsruhe in Germany and his colleagues is thought to contain 490 silver atoms linked by 188 sulphur atoms and 114 organic groups, $\mathrm{Ag}_{490} \mathrm{~S}_{188}\left(\mathrm{StC}_{5} \mathrm{H}_{11}\right)_{114 \cdot}$ " This is an idealized structure" based on energy calculations, explains Fenske, who published the structure this month (C. E. Anson et al. Angew. Chem. Int. Edn doi:10.1002/anie.200704249; 2008).

"With structures that size you are pushing the crystallography technique right to its limit," says chemist Paul Raithby at the University of Bath, UK. Yet, he adds: "As far as crystallography and mass spectrometry can prove anything, this structure is as definitively proved as you could get."

Fenske's molecules, described as 'clusters', produce crystals that are about 3 nanometres in diameter. The crystals have a welldefined outer 'shell' that is possible, though not simple, to characterize using $\mathrm{X}$-ray diffraction.

But delve beneath the crystals' outer shell and things get a lot more complicated. Rather than the regular structure seen in a typical crystal, Fenske's clusters contain disorder: a void, filled by silver and sulphur atoms linked together in haphazard disarray. Molecules of silver sulphide $\left(\mathrm{Ag}_{2} \mathrm{~S}\right)$ can be arranged in a number of different geometries, including cubic, octahedral or dodecahedral. The disorder inside Fenske's cluster is so great that he can't tell what the geometry is in any of his samples. He describes the interior as looking "molten".

The crystals have been tricky to define in more than one way: even though they grow in a crystalline way, the disordered core causes a problem. "If the interior of the particles is 'mobile' then they are not crystals," says Frank Leusen, a crystallographer at Bradford University, UK. And are they molecules? Some say yes, others are not so sure. "These systems are at the boundary between molecular chemistry and bulk materials chemistry," says Raithby. "I am at a bit of a loss finding an appropriate term," adds Leusen.

Because of their huge size, Fenske's clusters may have characteristics that transcend the limits of molecular chemistry and enter the realm of macroscopic particles. For example, they may have interesting electrical properties.

The sheer size and complexity of the clusters mean that their internal structure cannot be revealed using $X$-ray diffraction. The technique uses $X$-rays that are reflected off the atoms in a rotating crystal, creating patterns of spots. These spots are converted into a map of the electron density around the atoms, and eventually the molecule's structure is calculated. But with larger molecules the number of spots increases, the spots get closer together and can even overlap. Also the 